\title{
BMJ Open Prioritising outcomes for evaluating eosinophil-guided corticosteroid therapy among patients with acute COPD exacerbations requiring hospitalisation: a Delphi consensus study
}

\author{
Carey Meredith Suehs (10 , ${ }^{1}$ Maéva Zysman, ${ }^{2,3}$ Cécile Chenivesse, ${ }^{4}$ \\ Pierre-Régis Burgel, ${ }^{5} \mathrm{~F}$ Couturaud, ${ }^{6}$ Gaëtan Deslee, ${ }^{7}$ Patrick Berger, ${ }^{3,8}$ \\ Chantal Raherison, ${ }^{9}$ Gilles Devouassoux ${ }^{10}$ Christophe Brousse, ${ }^{11}$ Nicolas Roche,${ }^{5}$ \\ Mathieu Molimard, ${ }^{12}$ Thierry Chinet, ${ }^{13}$ Philippe Devillier, ${ }^{14}$ Pascal Chanez, ${ }^{15}$ \\ Romain Kessler, ${ }^{16}$ Alain Didier, ${ }^{17}$ Yan Martinat, ${ }^{18}$ Olivier Le Rouzic, ${ }^{4}$ A Bourdin ${ }^{1,19}$
}

To cite: Suehs CM, Zysman M, Chenivesse C, et al. Prioritising outcomes for evaluating eosinophilguided corticosteroid therapy among patients with acute COPD exacerbations requiring hospitalisation: a Delphi consensus study. BMJ Open 2020;10:e035811. doi:10.1136/ bmjopen-2019-035811

\section{- Prepublication history and} additional material for this paper are available online. To view these files, please visit the journal online (http://dx.doi. org/10.1136/bmjopen-2019035811).

Received 18 November 2019 Revised 17 March 2020 Accepted 04 June 2020

Check for updates

(C) Author(s) (or their employer(s)) 2020. Re-use permitted under CC BY-NC. No commercial re-use. See rights and permissions. Published by BMJ.

For numbered affiliations see end of article.

Correspondence to Carey Meredith Suehs; careysuehs@protonmail.com

\section{ABSTRACT}

Objectives Presently, those outcomes that should be prioritised for chronic obstructive pulmonary disease (COPD) exacerbation studies remain unclear. In order to coordinate multicentre studies on eosinophilia-driven corticosteroid therapy for patients hospitalised for acute exacerbation of COPD (AECOPD), we aimed to find consensus among experts in the domain regarding the prioritisation of outcomes.

Design A modified Delphi study was proposed to recognised COPD experts. Two brainstorming questionnaires were used to collect potential outcomes. Four subsequent rounds of questionnaires were used to rank items according to a six-point Likert scale for their importance in the protocol, as well as for being the primary outcome. Priority outcome criteria were predefined as those for which $\geq 70 \%$ of experts indicated that the outcome was essential for interpreting study results. Setting COPD exacerbation management in France. Participants 34 experts recommended by the French Language Pulmonology Society were invited to participate. Of the latter, 21 experts participated in brainstorming, and 19 participated in all four ranking rounds.

Results 105 outcomes were ranked. Two achieved consensus as candidate primary outcomes: (1) treatment failure defined as death from any cause or the need for intubation and mechanical ventilation, readmission because of COPD or intensification of pharmacologic therapy, and (2) the time required to meet predefined discharge criteria. The 10 secondary priority outcomes included survival, time with no sign of improvement, episodes of hospitalisation, exacerbation, pneumonia, mechanical or non-invasive ventilation and oxygen use, as well as comorbidities during the initial hospitalisation. Conclusions This Delphi consensus project generated and prioritised a great many outcomes, documenting current expert views concerning a diversity of COPD endpoints. Among the latter, 12 reached consensus as

\section{Strength and limitations of this study}

- Despite much recent enthusiasm in the domain, this is the first initiative addressing consensus for priority outcomes for eosinophil-guided corticosteroid therapy in acute exacerbation of chronic obstructive pulmonary disease (COPD) inpatients.

- Registration on the Open Science Framework was performed prior to data collection, thus ensuring methodological transparency.

- There was no expert attrition during the four rounds of ranking of 105 potential outcomes.

- Patient involvement was not integrated in the study design, and outcomes important to patients may therefore be lacking.

- The presented priority outcomes have the advantage of covering both short and longer term time frames, being objective in nature and not associated with added costs beyond careful data recording. They are furthermore applicable to all COPD exacerbation studies.

priority outcomes for evaluating the efficacy of eosinophildriven corticosteroid therapy in AECOPD inpatients.

Study registration The eo-Delphi project/protocol was registered on 23 January 2018 at https://osf.io/4ahqw/.

\section{BACKGROUND}

In chronic obstructive pulmonary disease (COPD), there is no established biomarker that strongly correlates with inflammatory or degenerative processes affecting lung tissue or treatment responses. ${ }^{12}$ Traditionally, the forced expiratory volume in $1 \mathrm{~s}$ from spirometry is used as a key monitoring tool. ${ }^{3}$ 
However, pulmonary function measures can be highly variable, and repeated measures are often necessary in order to reinforce confidence that change with time is occurring. ${ }^{2}$ They also often demonstrate no correlation with patient reported outcomes or symptoms and cannot be considered as a surrogate for the latter. ${ }^{24}$ Furthermore, many COPD outcomes, such as dyspnoea, exacerbation rates, physical or exercise capacities or general health status have no gold standard. Finally, the comorbidities that often accompany COPD can significantly influence outcomes and generate population heterogeneity. ${ }^{1}$ This is all-the-more true in the context of severe exacerbations requiring hospital admission where multiple factors from multiple fields have the potential to influence the course and subsequently the outcomes of the disease. Given this context, and despite several reviews, the development of diverse patientreported outcomes ${ }^{45}$ and the formation of task groups, ${ }^{2}$ those outcomes that should be prioritised for COPD exacerbation studies remain unclear.

As demonstrated above, the choice of outcomes that should be monitored in order to describe treatment responses among COPD patients is clearly a subject of current debate. In addition, how outcomes are chosen, and why, is a key point in drug trial design. When views are too narrow, the selected outcomes might apply to only one particular group's interests or needs. ${ }^{6}$ Often, less favourable, difficult-to-assess or heterogeneous outcomes are neglected. A literature review ${ }^{2}$ found an impressive number of extremely different main and secondary outcomes that have been assessed in acute exacerbations of COPD (AECOPD). Interestingly, outcomes often differ between admitting and non-admitting AECOPD. In admitted patients, they also differ according to intensive care unit (ICU) requirements, but many other modifiers are cited, reflecting the varying definitions for COPD exacerbation and heterogeneity in management practices among sites and/or countries. In light of the lack of current consensus on COPD outcomes as well as to avoid the latter problems, we aimed to use the Delphi process as a means to select a priority outcome set for future COPD drug trials evaluating the effects of corticosteroid therapy within blood eosinophil strata. The results are expected to provide main and secondary outcomes that share the following properties: (i) to specifically address the question being asked, to be free from the bias associated with (ii) commercial interests, or from (iii) potentially interfering factors (comorbidities, social issues and departmental singularities) and finally to be feasible, shareable, understandable and applicable to everyday practice. Most importantly, Delphi processes build consensus that can be recognised by physicians and policy/guideline makers in the field, which is crucial for investigator adoption of a research protocol. Finally, the Delphi process has the added advantages of being anonymous (which avoids dominance by opinion leaders and peer pressure) and applicable to a relatively large number of individuals more efficiently than work groups. ${ }^{6}$
Currently, there is a gap in the literature concerning corticosteroid therapy response among COPD inpatients as a function of blood eosinophil levels. ${ }^{7-9}$ The eo-Drive trial (https://osf.io/9j7uk/) seeks to fill this gap by comparing eosinophilic COPD patients hospitalised for acute exacerbation and treated via systemic corticosteroid therapy with a similar population treated via placebo. Secondarily, the same comparison will be made among non-eosinophilic COPD patients. Within this context, the objective of the present work was to use the Delphi technique among a panel of experts to reach consensus for defining priority outcomes for future COPD drug trials evaluating the effects of corticosteroid therapy within blood eosinophil strata. Though focused on a specific domain, the knowledge generated by this exercise provides a study-design-aid likely applicable to any COPD treatment efficacy settings involving exacerbating populations.

\section{METHODS}

\section{Overview of the Delphi method}

A series of anonymous, electronically administered (www. surveymonkey.com) questionnaires was sent to a panel of experts from the COPD working group at the SPLF (the Société de Pneumologie de Langue Française (the French Language Pulmonology Society)), thus ensuring appropriate knowledge/experience regarding the problem to be addressed (figure 1). Each round of questionnaires results in a corpus of information that was fed back to the panel before performing the subsequent round. The first two 'brainstorming' questionnaires represent an 'itemcollection' stage and were open in nature (see below). The subsequent rounds of 'ranking' questionnaires were used to prioritise items according to their importance for appearing in the protocol, as well as for being a candidate primary outcome. Individual outcome ranking continued for a maximum of four rounds, or until dropping criteria were achieved. A designated study facilitator (CMS) organised questionnaire logistics, feedback to participants, data collection, analysis and writing.

This volunteer-based Delphi study using anonymous questionnaires is categorised as not falling under the jurisdiction of the 'Jardé Law' in France.

\section{Patient and public involvement}

There was no patient or public involvement in this study.

\section{The expert panel}

French clinicians who were members of the COPD working group at the SPLF and who had previously published in the domain or who had included COPD patients in previous studies were asked to participate. The participation list was prepared by the Société de Pneumologie de Langue Française (the French Language Pulmonology Society) and not distributed to members in order to maximise anonymity. ${ }^{6}$ 


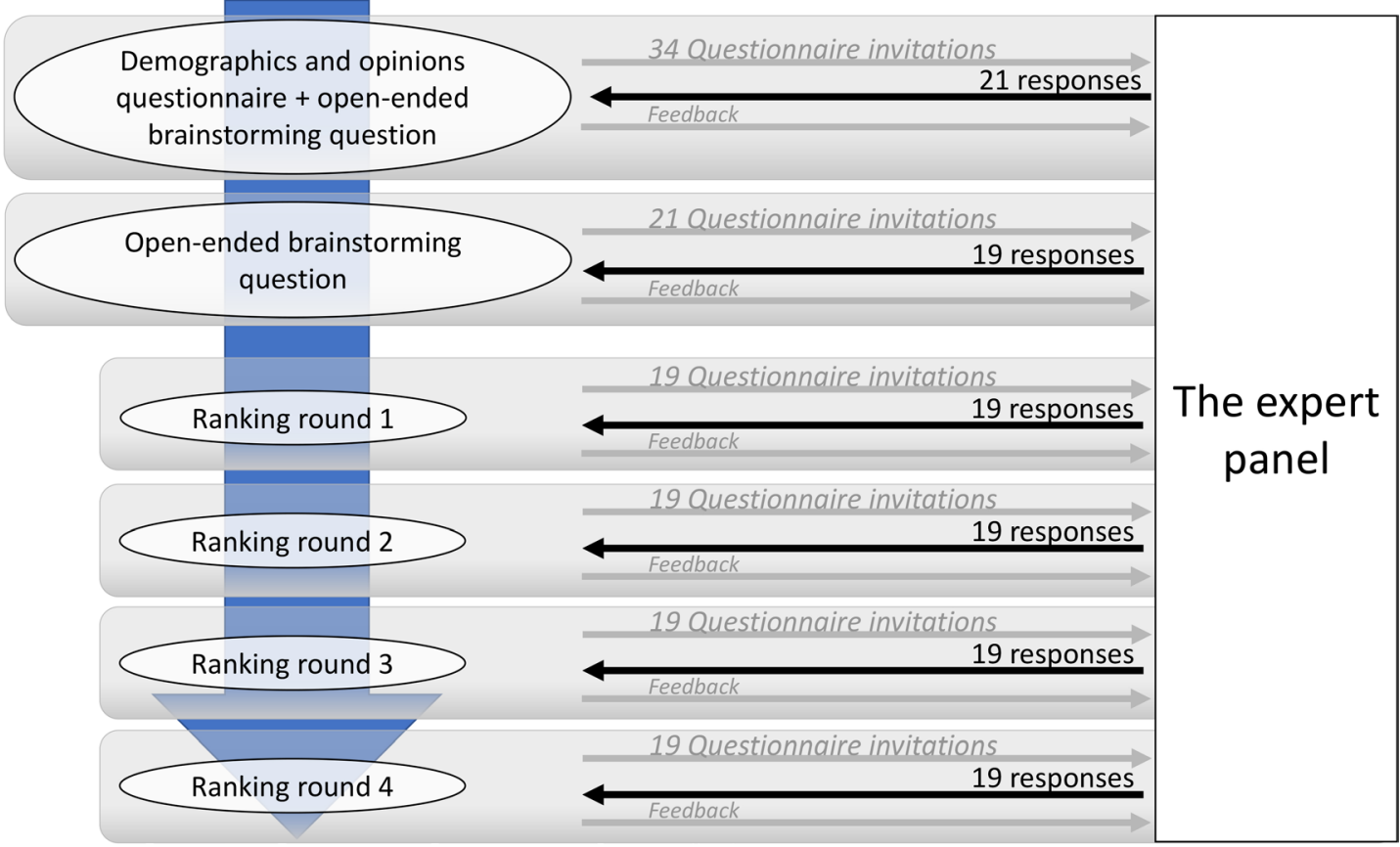

Figure 1 Methodology flow chart for the eo-Delphi project. The expert panel was invited to participate in six sequentially administered questionnaires. Only those experts who responded to an invitation were subsequently invited to participate in the next questionnaire. The first questionnaire included questions describing the demographics of the expert panel, as well as opinion-type questions. It ended with an open-ended brainstorming question. The second questionnaire corresponded to a second round of brainstorming. The final four questionnaires were ranking questionnaires, where outcomes proposed by the expert panel during the brainstorming process were evaluated according to a Likert scale specifically designed for prioritising outcomes in research trials (table 1).

\section{Demographics and opinion questionnaire}

The round 1 instrument started with an online demographic $^{8}$ and opinion questionnaire (prepared by the eo-Drive organising team) (figure 1) that captured data for the following variables: age, sex, academic or private practice, current position, years of experience in COPD management, and contributes to the development of local exacerbation management policy (yes/no). In addition, the experts were asked how familiar they were with GOLD and French national guidelines questions, and then to use a visual analogue scale (VAS) to quantify how often they applied a given guideline (VAS ranging from 0 (never) to 100 (all the time)) in their practice and then how they would rate that guideline (VAS ranging from 0 (completely inadequate) to 100 (perfect)). Experts were also asked how they rated their local hospital discharge criteria (VAS ranging from 0 (completely inadequate) to 100 (perfect)). Additional organisational questions beyond the scope of the present outcome summary paper were presented, the content of and responses to which are summarised in online supplementary additional file 1. Questionnaires and related study documents are available on the eo-Delphi study registration site: https://osf.io/8f3aj/

\section{Brainstorming questionnaires}

The round 1 instrument ended by asking participants to upload responses to the following question: 'Which outcomes should be recorded in order to best evaluate treatment strategy efficacy, as well as informative disease monitoring, for the eo-Drive study?' An openended question was chosen as the least-biassed means of representing the diverse opinions ${ }^{6}$ likely present among experts concerning how best to measure the success of corticosteroids for the treatment of COPD exacerbations.

The first round of open-ended responses was edited for redundancy, English language and homogenisation of time frames, grouped by theme or domain, and then provided to all participants. A second round of brainstorming was then performed to allow for new ideas triggered by the first round (figure 1). Responses from the second round were edited in the same way as the first round, and then provided to all participants.

\section{Item-rating questionnaires}

Following the brainstorming process, each outcome was separately evaluated by each participant via successive item-rating questionnaires (figure 1). In each of the latter, participants were asked to prioritise the importance of each outcome by applying a six-point Likert-type scale (table 1). At the end of each round, results were distributed to participants. Additionally, the previous ranking round's results were embedded within the subsequent questionnaire so that participants were sure to have them on hand when ranking. 


\begin{tabular}{ll} 
Table 1 & The Likert scale used for prioritising outcomes \\
Score & Likert item \\
\hline 1 & This outcome is of no importance or off topic; it should be excluded from the protocol. \\
2 & This outcome is of dubious value; there are minimal reasons for including it in the protocol as a secondary outcome. \\
3 & $\begin{array}{l}\text { This outcome is of moderate importance. It should be included in the protocol as a secondary outcome if logistically } \\
\text { easy to do so. }\end{array}$ \\
& $\begin{array}{l}\text { This is an important though non-essential outcome. It has high scientific value. Effort should be made to include it in } \\
\text { the protocol as a secondary outcome. }\end{array}$ \\
This is an essential outcome without which our capacity to respond to or interpret the study's primary objective will be \\
compromised; it must be included in the protocol as a secondary outcome. \\
This is an essential outcome without which our capacity to respond to or interpret the study's primary objective will be \\
compromised; it must be included in the protocol as a candidate primary outcome.
\end{tabular}

\section{Criteria for dropping outcomes at the end of each round of item-rating}

Outcomes that met at least one of the following criteria were not represented for item-rating in a subsequent round:

- $\geq 70 \%$ of participants indicate the same score.

- $\geq 70 \%$ of participants indicate a score $\leq 2$.

\section{The definition of consensus and outcome classifications}

Consensus is defined by a $\geq 70 \%$ agreement between Delphi participants. This threshold was agreed on prior to study commencement because it represents an easy-tocalculate, attainable and strong majority vote. As defined in our preregistered protocol (https://osf.io/4ahqw/), priority outcomes are specifically defined as those for which $\geq 70 \%$ of participants indicate a score $\geq 5$. The reader should note that this definition allows for $30 \%$ dissensus among experts in regard to what can be considered as a 'priority' outcome. We additionally define the following classes of outcomes:

- Important: $\geq 70 \%$ of participants gave a score $\geq 4$ (excepting those classed as priority outcomes).

- Non-essential secondary: $\geq 70 \%$ of participants gave a score of $\geq 3$ (excepting those classed as priority or important outcomes)

- Lacking importance: $\geq 70 \%$ of participants gave a score $\leq 3$ (excepting those classed as non-essential secondary or excluded outcomes).

- Excluded: $\geq 70 \%$ of participants gave a score $\leq 2$.

- Controversial: those outcomes not falling into one of the previous classes.

\section{Information provided to participants before round 1}

The facilitator contacted each invited participant before round 1 . The goal was to raise awareness for the study. Investigators are busy, and likely discard most surveys. Individual contact was initiated to help avoid this kind of problem. The facilitator also provided information concerning the study rational, design (ie, the study protocol) and associated articles for (non-obligatory) background reading to each participant.

\section{Minimising participant attrition}

As consensus forms, participants with minority opinions can become discouraged and tend to drop out of the process, resulting in an overestimation of consensus. ${ }^{6}$ To avoid this, only participants who completed the final questionnaire (and therefore all questionnaires) were eligible for authorship. ${ }^{6}$ Persons who did not respond to a questionnaire despite the best efforts of the facilitator to encourage participation were not solicited for the next round of questionnaires.

\section{Descriptive statistics}

Statistical analysis was performed using the $\mathrm{R}$ programming environment V.3.3.0 (R Core Team 2016). Centrality statistics for quantitative demographic variables (table 2) with normal distributions according to Shapiro-Wilks tests are given as means $\pm \mathrm{SD}$. Other quantitative variables (VAS scores) are summarised as medians followed by the associated IQR, and qualitative ones as numbers and percentages. Prioritised outcomes were ordered according to their final mean rank.

\section{RESULTS \\ Participation}

Between 23 January and 16 February 2018, 21 out of 34 invited COPD experts completed the first demographics/opinions questionnaire and provided a list of outcomes that they would like to see evaluated by their peers (figure 1). The remaining 13 failed to respond to multiple participation requests from the study facilitator. The participants were on average $50.67 \pm 8.16$ years old with over 20 years of experience in COPD management and the vast majority were men working in a University Hospital; $71.43 \%$ of participants had achieved professorship (table 2).

Nineteen out of 21 experts (two did not respond to multiple invitations to continue to the next round) further completed brainstorming round 2 and subsequent minor additions were made to the list of outcomes. The same 19 experts participated in ranking rounds 1 to 
Table 2 Descriptive statistics for eo-Delphi participants

\begin{tabular}{|lcl} 
& $\begin{array}{l}\text { Completed brainstorming round 1 } \\
\mathbf{n = 2 1}\end{array}$ & $\begin{array}{l}\text { Completed brainstorming round } \mathbf{2} \\
\text { and ranking rounds 1-4 } \\
\mathbf{n}=\mathbf{1 9}\end{array}$ \\
\hline Age (years) & $50.67 \pm 8.16$ & $51.05 \pm 8.51$ \\
\hline Gender: male & $18(85.71 \%)$ & $16(84.21 \%)$ \\
\hline University Hospital & $20(95.24 \%)$ & $18(94.74 \%)$ \\
\hline Professor & $15(71.43 \%)$ & $13(68.42 \%)$ \\
\hline Assistant professor & $1(4.76 \%)$ & $1(5.26 \%)$ \\
\hline Research MD & $1(4.76 \%)$ & $1(5.26 \%)$ \\
\hline Head of department & $8(38.10 \%)$ & $8(42.11 \%)$ \\
\hline Head of group of departments & $1(4.76 \%)$ & $1(5.26 \%)$ \\
\hline Scientist (PhD) & $1(4.76 \%)$ & $1(5.26 \%)$ \\
\hline Part-time with private practice & $1(4.76 \%)$ & $1(5.26 \%)$ \\
\hline Private practice only & $1(4.76 \%)$ & $1(5.26 \%)$ \\
\hline Years of experience in COPD management & $22.00 \pm 8.26$ & $22.26 \pm 8.62$ \\
\hline Helps develop local exacerbation & $6(28.57 \%)$ & $5(26.32 \%)$ \\
\hline management policy & & \\
\hline
\end{tabular}

COPD, chronic obstructive pulmonary disease.

4 (figure 1), which ended on 7 March, 29 March, 27 April and 4 July 2018 , respectively.

The research experience of the 19 final participants was high, with $84.20 \%$ indicating research positions $(73.68 \%$ university professors or assistant professors, plus one research MD and one scientist). Nearly half (47.37\%) were in leadership positions (head of department) in their respective hospitals, but only $26 \%$ indicated that they were involved in the development of local exacerbation management policy. Their average number of

A

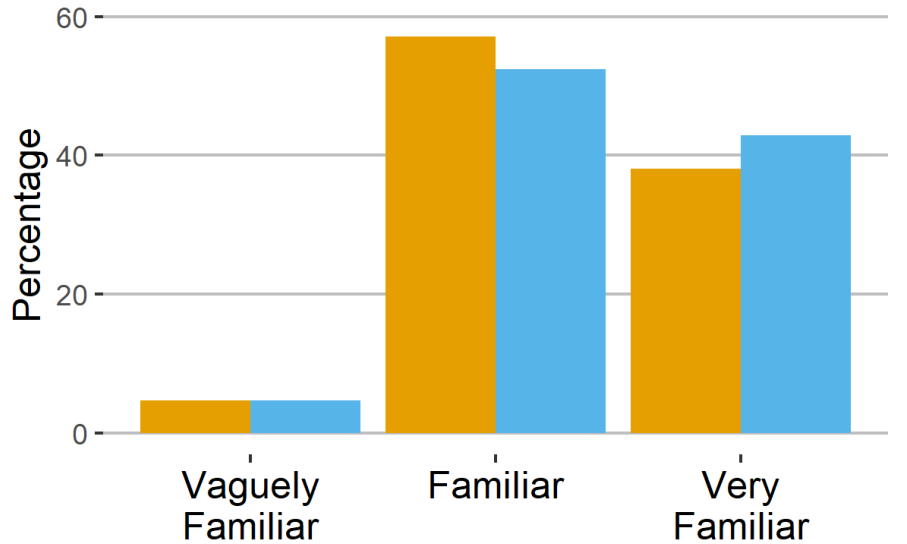

years of COPD management experience was 22.26 \pm 8.62 , further reinforcing the high level of appropriate expertise for the group.

\section{Expert opinions concerning current guidelines and local hospital discharge criteria}

As concerns current guidelines, over $95 \%$ of the expert panel indicated that they were either familiar or very familiar with both GOLD and French (SPLF) guidelines (see figure 2A). VAS scores indicate that experts apply

\section{B}

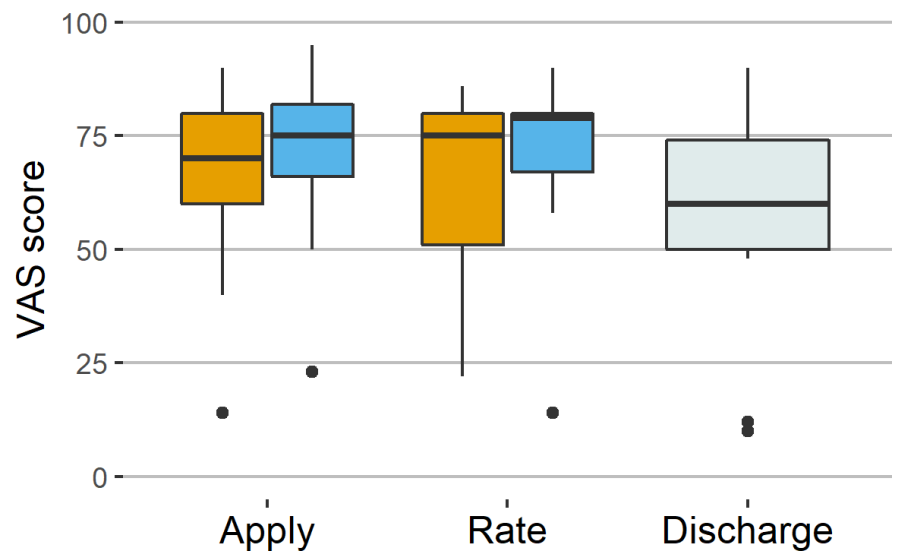

Figure 2 COPD expert opinions concerning current guidelines (GOLD in gold and French national guidelines (SPLF) in blue) as well as local hospital discharge criteria (light grey). Panel A: \%Response rates when asked how familiar experts were with a given guideline. Panel B: Visual analogue scale scores for how often experts applied a given guideline (ranging from 0 (never) to 100 (all the time)) in their practice and then how they would rate that guideline (score ranging from 0 (completely inadequate) to 100 (perfect)). Experts were also asked how they rated their local hospital discharge criteria (score ranging from 0 (completely inadequate) to 100 (perfect)). Medians are presented as horizontal lines, first to third quartiles as boxes, $1.5 \times$ the IQR as whiskers and outliers as points. COPD, chronic obstructive pulmonary disease; SPLF, the Société de Pneumologie de Langue Française. 
guidelines a majority of the time, but not always. They generally rate guidelines quite well, with slightly higher ratings for French guidelines compared with GOLD (see figure 2B). In comparison, experts rated their local hospital discharge criteria at a median score of 60 (IQR of 50-74; see figure 2B), suggesting some room for improvement.

\section{Outcomes}

Raw and summarised outcome lists resulting from brainstorming rounds 1 and 2 are available at the study registration site: https://osf.io/8f3aj/. Among the suggested outcomes raised during brainstorming, we set aside those that were obviously baseline characteristics; the latter sort were noted, but not subjected to prioritisation. Many outcomes were of a type associated with survival or recurrent event analysis (eg, time to first exacerbation, days alive and free from hospitalisation, exacerbation frequency), and data collection would have involved recording the beginning and end dates/times for an event (or a series of events, like exacerbations or hospitalisations). The latter types of outcomes were grouped together under the label 'event tracking', since they share common data set structures.

The final ranking round results are presented in online supplementary additional file 2, arranged from highest mean ranking to lowest mean ranking. Some of the proposed outcomes are quite specific, and the reader is encouraged to refer to online supplementary additional file 2 for outcome details.

\section{Priority outcomes}

Priority outcomes are considered by a strong majority ( $\geq 70 \%)$ of Delphi participants to be essential (table 1). Their absence from eo-Drive would compromise the investigators' capacity to respond to or interpret the study's primary objective (therapeutic efficacy of corticosteroids for the treatment of inpatient COPD exacerbation). The priority outcome set for the eo-Drive study is presented in table 3. Two achieved consensus as candidates for a primary outcome: (1) treatment failure according to Niewoehner et $a l,{ }^{10}$ which was the outcome with the highest mean rank (mean rank of 5.68, online supplementary additional file 2), and (2) the time required to meet predefined discharge criteria during the initial hospitalisation (mean rank of 5.58, online supplementary additional file 2). Ranking scores for survival were split between primary versus secondary essential outcome status, and the remaining priority outcomes reached consensus as essential secondary outcomes.

\section{DISCUSSION}

In this six-round (two rounds of brainstorming for generating an outcome list plus four rounds of outcome ranking) Delphi process involving 21 COPD experts and representing 11 University hospitals, one privatenot-for-profit hospital and two private medical centres, consensus was achieved for a priority outcome set for the evaluation of eosinophil-guided corticosteroid therapy in patients hospitalised for a COPD exacerbation. Given the increasing interest in eosinophil-driven treatment for COPD patients, and the fact that COPD management guidelines currently call for further prospective trials with eosinophil stratification to widen the appropriate evidence base, ${ }^{78}$ this consensus is timely. The resulting priority outcome set is also applicable to other COPD exacerbation studies outside the eosinophil-related context.

Participation rates from the highly experienced panel were excellent, with a $100 \%$ retention rate throughout the ranking process. Of note, 105 outcomes were proposed for prioritisation, and this generous brainstorming component demonstrates the importance of conducting Delphi exercises in order to maximise the likelihood for a multicentre clinical trial to appropriately and accurately answer a precise therapeutic question while meeting the expectations of multiple researchers. Finally, a subset of 12 proposed outcomes reached consensus among the expert panel as priority outcomes without which the evaluation of the therapeutic efficacy of eosinophil-driven corticosteroid therapy for severe AECOPD was considered as compromised (see table 3 ).

The highest-ranking priority-outcome in the present study is a composite-measure of treatment failure (table 3). Treatment failure is also the first critical outcome indicated by a European Respiratory Society/American Thoracic Society (ERS/ATS) task force providing guidelines on COPD exacerbation management and addressing questions on corticosteroid therapy efficacy in out-patient (general efficacy) and in-patient (intravenous vs oral corticosteroids) contexts. ${ }^{9}$ This choice of primary outcome is therefore well poised for impacting future guidelines and practice. In addition, approximately $1 / 3$ of previously published clinical trials on COPD exacerbation report results for treatment failure, ${ }^{8}$ thus assuring a high degree of comparability with previous studies. Specific, recently influential examples include studies by Bafadhel et al, first comparing eosinophil-driven to systematic corticosteroid therapy in ambulatory patients (with treatment failure differences of $5 \%$ vs $13 \%$, respectively), ${ }^{11}$ and second a letter ${ }^{12}$ describing a $55 \%$ difference in treatment failure rates when retrospectively comparing corticosteroid therapy versus placebo among patients with higher eosinophil counts.

Care must be taken, however, when defining the composite elements of treatment failure, as considerable variation in definitions has been noted..$^{13}$ The composite measure of treatment failure proposed by the ERS/ATS task force included death, admission to the ICU, readmission to the ICU due to COPD or intensification of pharmacological therapy. ${ }^{9}$ The latter is extremely close to the definition of treatment failure retained by the present paper (table 3) and first defined by Niewoehner et al, ${ }^{10}$ easily allowing for integration into future task force initiatives. The eo-Drive priority outcome set also provides for 


\section{Priority outcome}

Final mean

score

$\%$ Consensus

Treatment failure (according to Niewoehner et $\mathrm{al}^{10}$ ) defined as death from any cause or the need for intubation and mechanical ventilation, readmission because of COPD or intensification of pharmacologic therapy†

The time required to meet predefined dischargeability criteria** $\left(\mathrm{DC}_{\mathrm{time}}\right)$ during the initial hospitalisation $\dagger \dagger \dagger$

\begin{tabular}{|c|c|c|}
\hline Survivalł & 5.21 & 89.47 \\
\hline $\begin{array}{l}\text { Treatment failure defined as the need to start or repeat treatment within } 30 \text { days of } \\
\text { randomisation§†† }\end{array}$ & 5.00 & 84.21 \\
\hline Episodes* of oxygen use§†† & 4.95 & 89.47 \\
\hline Episodes ${ }^{\star}$ of non-invasive ventilation§†† & 4.84 & 84.21 \\
\hline $\begin{array}{l}\text { Episodes }{ }^{\star} \text { of exacerbation (distinguishing mild to moderate exacerbations (requiring } \\
\text { antibiotics and/or de novo/increased oral steroids) and severe exacerbations (requiring } \\
\text { hospitalisation or emergency room services))§†† }\end{array}$ & 4.74 & 84.21 \\
\hline Episodes* of pneumoniaß†† & 4.68 & 84.21 \\
\hline Hospital stays* (distinguishing intensive care, intermediate care and ward stays)§†† & 4.63 & 73.68 \\
\hline
\end{tabular}

At least $70 \%$ of eo-Delphi participants gave a Likert score of 5 (essential secondary outcome) or 6 (essential primary outcome) for each listed outcome (\%Consensus).

*Episodic outcomes require the recording of the start and finish date for each episode observed throughout the study.

†Consensus as candidate primary priority outcomes.

‡Survival scores were split between primary and secondary priority outcome status.

$\S$ Consensus as secondary priority outcomes.

१Niewoehner et a $\left.\right|^{10}$ define intensification of pharmacologic therapy as the prescription of open-label systemic glucocorticoids, high-dose inhaled glucocorticoids (more than eight puffs per day oftriamcinolone acetonide or its equivalent), theophylline or any combination ofthese three therapies.

${ }^{* *}$ The predefined discharge ability criteria in eo-Drive are: acidosis has normalised; symptoms have improved to a level compatible with discharge; capable of performing minimal daily living activities. The earliest occurring of the latter will be used to determine $\mathrm{SI}_{\text {time }}$ ††These outcomes can be biased by competing risks, namely mortality. Care should therefore be taken when aggregating them, and the complement (eg days alive and without the outcome in question) thus considered to avoid this bias. Alternatively, competing-risk analyses may be required.

COPD, chronic obstructive pulmonary disease.

a simpler treatment failure definition based uniquely on treatment recurrence as a secondary outcome (table 3); this will enable comparisons with national database prescription information in France where the future trial will take place (https://osf.io/8f3aj/). In addition to the individual elements composing a composite measure of treatment failure, their use should also be juxtaposed with how patients' preferences in terms of end-of-treatment options are locally taken into account. The latter may include choices that result in the non-transfer to an ICU or a lack of mechanical ventilation (eg), even though one or both were indicated.

The previously mentioned ERS/ATS task force further indicated mortality, hospital readmission, length of stay (LOS) and time to next exacerbation as critical outcomes (with adverse events as an 'important' outcome) for guiding treatment recommendations in an inpatient context (in their case, comparing intravenous and oral corticosteroid therapies). ${ }^{9}$ In comparison, the priority outcome set selected during the present study incorporate all the previous, and further requires additional information. For example, within an initial hospitalisation time frame, time to signs of improvement $\left(\mathrm{SI}_{\text {time }}\right)$ and dischargeability $\left(\mathrm{DC}_{\text {time }}\right)$ were considered as essential outcomes, rather than just LOS. In a simple scenario, once a patient improves and meets discharge criteria, he/she can theoretically leave the hospital, and $\mathrm{DC}_{\text {time }}$ should approach LOS. However, in many domains, meeting predefined discharge criteria does not always lead to actual hospital discharge due to interference from comorbidities, social issues, the availability of appropriate rehabilitation or care facilities $^{14}$ or individual hospital cluster effects. ${ }^{15}$ The proposed $\mathrm{DC}_{\text {time }}$ variable should therefore have fewer sources of heterogeneity as compared with LOS (though it also requires more data capture). Finally, immediate-timeframe outcomes may be particularly short for eosinophilic groups receiving corticosteroid therapy as compared with non-eosinophilic or placebo groups. ${ }^{16}$ It is not surprising 
then that the eo-Delphi expert panel indicated $\mathrm{DC}_{\text {time }}$ as a second candidate primary outcome for future studies on eosinophil-driven corticosteroid therapy.

A limitation of the present study is the lack of patient involvement during study design and deployment. The presented outcomes therefore represent the physicians' point of view only, and outcomes important to patients may be lacking. The definition of consensus also allowed for up to $30 \%$ dissensus when classifying outcomes, and the reader is thus encouraged to refer to online supplementary additional file 2 as the distribution of dissensus votes over ranks can be quite variable and informative. A further limitation of this study is the lack of a literature review for gathering further outcomes.

A major methodological consideration that should be given thought when using the outcomes in table 3 is the potential for competing risks, namely mortality. To avoid this source of bias, variables of the type 'days alive and free from hospitalisation', which take the complement of time spent in a given event, can be used when aggregating episode-type or duration-type data. This effectively replaces mortality-affected measures with low values and avoids the related competing risk bias. Alternatively (especially when the latter is not possible), competing risk analyses can be employed during a sensitivity evaluation (as classically performed for survival-type analyses) to explore potential impacts on results.

The reader should also note that specific time frames are not consistently enforced as a result of this outcomeprioritising Delphi study, which was performed early-on during a study-design process. Because the future eo-Drive study has a follow-up time of 3 months, the latter is the suggested time frame unless otherwise specified. Because time frame variation is a source of heterogeneity in the literature, study designers should carefully choose time frames to maximise clinical validity and comparisons with past studies. Further guidance on time frames is also likely upcoming, with the anticipated development of a core outcome set for AECOPD (http:/ / www.comet-initiative.org/Studies/Details/1325).

Despite the above limitations, the presented priority outcome set has the advantages of being objective in nature, applicable to any COPD exacerbation study and not associated with added costs beyond careful data recording. Specifically, obtaining high-quality survival or episode data requires effort by data-capture teams and maintained contact with patients and/or their care providers. These data types can give rise to numerous useful measures, such as 'time to next event', mean cumulative functions for recurrent events ${ }^{17}$ and event numbers/rates. The most recent eosinophil-driven corticosteroid therapy study in COPD by Sivapalan et al used the number of days alive and out of hospital within 14 days after recruitment as their primary outcome. ${ }^{16}$ The hospital episode data collected during eo-Drive will provide the means for a comparison with the latter by using each day a patient is alive and out of the hospital as a cumulative recurrent event plotted against time. ${ }^{17}$
Interestingly, though asked to consider an eosinophilguided corticoid therapy context, simultaneous changes in blood eosinophilia did not reach consensus as a priority outcome, but were classified as non-essential secondary outcomes (online supplementary additional file 2), suggesting that changes in this biomarker were not considered as essential for interpretation of results. This may be because experts were strictly considering the evaluation of treatment efficacy, without exploring associated potential mechanisms. It may also suggest that certain experts do not consider eosinophilia to be a pertinent mechanism of disease for many COPD patients.

\section{Conclusions}

In conclusion, if given the opportunity, collaborating investigators will provide a plethora of information concerning outcome expectations for a given clinical research project. In the present study, the Delphi process proved efficient for drawing consensus and team federation for the project. The resulting set of 12 priority outcomes appear logical, objective, feasible and reproducible vis-à-vis efficacy testing for eosinophil-driven corticosteroid therapy strategies in patients hospitalised for AECOPD, and furthermore applicable to COPD exacerbation studies in general. Finally, the ranking exercise documents current knowledge among investigators concerning a wide range of outcomes, providing a valuable tool for COPD study designers and students of methodology.

\section{Author affiliations}

${ }^{1}$ Maladies Respiratoires, Univ Montpellier, CHU Montpellier, Montpellier, France ${ }^{2}$ Maladies Respiratoires, Pôle Cardiothoracique, CHU Haut-Lévèque, Bordeaux, France

${ }^{3}$ Centre de Recherche Cardio-thoracique de Bordeaux, INSERM U1045, Univ. Bordeaux, Bordeaux, France

${ }^{4}$ Pneumologie et Immuno-Allergologie, CHU Lille, Univ. Lille, CNRS, Inserm, Institut Pasteur de Lille, U1019 - UMR 8204 - CIIL - Center for Infection and Immunity of Lille, Lille, France

${ }^{5}$ Respiratory Medicine, Cochin Hospital (APHP), University Paris Descartes (INSERM U1016 Institut Cochin), Université de Paris, Paris, France

${ }^{6}$ Internal Medicine and Chest Diseases, EA3878, CIC-INSERM1412, Brest University Hospital Centre, Brest University, Brest, France

${ }^{7}$ Pulmonary Medicine, INSERM U1250, University Hospital of Reims, Reims, France ${ }^{8}$ Exploration Fonctionnelle Respiratoire, CIC 1401, CHU Bordeaux, Bordeaux, France ${ }^{9}$ Bordeaux Population Health Research Center, U1219 BPH - Inserm - Université de Bordeaux, Bordeaux, France

${ }^{10}$ Pneumologie, Hôpital de la Croix Rousse, Hospices Civils de Lyon et Université Claude Bernard Lyon 1, EA7426, Lyon, France

${ }^{11}$ Clinique du Parc, 34170 Castelnau le Lez, France

${ }^{12}$ Pharmacologie, Univ. Bordeaux, INSERM U1219, Bordeaux, France

${ }^{13}$ Pneumologie et Oncologie Thoracique, CHU Ambroise Paré, AP-HP, Université de Versailles SQY, Saint-Quentin-en-Yvelines, France

${ }^{14}$ Airway Diseases, UPRES EA 220, Foch hospital, University Paris-Saclay, Suresnes, France

${ }^{15}$ Respiratory Diseases, AP-HM, INSERM, INRA, C2VN Aix Marseille Université, Marseille, France

${ }^{16}$ Pneumologie, Fédération de médecine translationnelle, Université de Strasbourg, Strasbourg, France

${ }^{17}$ Pôle des Voies Respiratoires, Hôpital Larrey, CHU de Toulouse et Université Paul Sabatier, Toulouse, France

${ }^{18}$ CM PAROT, Lyon, France

${ }^{19}$ PhyMedExp, Univ Montpellier, INSERM, CNRS, CHU Montpellier, Montpellier, France 


\section{Twitter Carey Meredith Suehs @SuehsCarey}

Contributors $A B$ originated the need for a Delphi process to select priority outcomes for the eo-Drive study. CMS wrote the eo-Delphi protocol and designed the methodology, ranking scale and outcome classification system. She also implemented the Delphi process, performed data collection, data wrangling, statistics, graphics and writing of the study report. All authors except CMS (MZ, CC, PRB, FC, GaD, PB, CR, GiD, CB, NR, MM, TC, PD, PC, RK, AD, YM, OLR and AB) completed the entire Delphi process. CMS and $A B$ wrote the first draft of the article manuscript. All authors read and participated in the correction of the manuscript, critical input and approved the final version.

Funding The authors have not declared a specific grant for this research from any funding agency in the public, commercial or not-for-profit sectors.

Competing interests As a consensus-building project among COPD experts, there are no specific conflicts of interest for this study. For thoroughness, past collaborations with industry are presented here. $\mathrm{AB}$, as an advisory board member, congress participant or study investigator, has previously received honoraria, grants or other forms of support from Astra Zeneca, GSK, Boeringher Ingelheim, Novartis, Teva, Regeneron, Chiesi Farmaceuticals, Actelion, Gilead and Roche. AD reports past collaborations (affiliation or financial interest or interest of any kind in the past 3 years) with AstraZeneca, GSK, Chiesi, Circassia, ALK, Novartis, Pierre Fabre, Boehringer-Ingelheim, Menarini, Teva, Zambon. CC reports interventions, expertise, grants or congress support from ALK-Abello, AstraZeneca, Boehringer Ingelheim, Chiesi, GlaxoSmithKlein, MEDA Pharma, Medexact, Novartis, Pierre Fabre, Pfizer, Roche, Sanofi, Santelys, TEVA. CR reports board participation, symposiums and congress fees for Astra Zeneca, Boeringher Ingelheim, Chiesi, GSK, Novartis. FC reports having received research grant support from Pfizer and Bristol-Myers Squibb, and fees for board memberships or symposia from Bayer, Bristol-Myers Squibb/Pfizer and Astra Zeneca and having received travel support from Bayer, Bristol-Myers Squibb/Pfizer, Daiichi Sankyo, Boehringer Ingelheim, Leo Pharma, Intermune and Actelion. GaD reports personal fees from Astra Zeneca, Boehringer Ingelheim, Chiesi, Novartis, Nuvaira, BTG/PneumRx. GeD declares being a consultant for Novartis Pharma, Astra-Zeneca, GSK, Boehringer Ingelheim, Mundi Pharma, Vivisol, TEVA, ALK, SANOFI, Menarini, participating at medical meetings for GSK, Astra-Zeneca, Novartis Pharma, Chiesi, MSD, Takeda, AGIR adom, Orkyn, TEVA, Mundi Pharma, ALK, Stallergène, SANOFI, taking part in clinical trails for (investigator)—GSK, ALK, Novartis Pharma, Boehringer-Ingelheim, Vitalair, AB Science, Amgen, Lilly, Astra Zeneca, Sanofi, Roche, TEVA, research fellowshipGSK, Novartis Pharma, MSD, Chiesi; MZ reports grants and personal fees from Novartis, personal fees and non-financial support from Boehringer Ingelheim, personal fees and non financial support from Astra Zeneca, personal fees from Chiesi, personal fees from GSK outside the submitted work. NR reports grants and personal fees from Boehringer Ingelheim, Novartis, Pfizer and personal fees from Teva, GSK, AstraZeneca, Chiesi, Mundipharma, Cipla, Sanofi, Sandoz, 3M, Trudell, Zambon. OLR reports personal fees and non-financial support from AstraZeneca, Boehringer Ingelheim, Chiesi, Lilly and Novartis; non-financial support from ALK, GlaxoSmithKlein, ICOMED, LEO Pharma, MSD, MundiPharma, Pfizer and TEVA. Outside the submitted work, PB declares grants from GSK, Novartis, Boehringer, AstraZeneca, Chiesi; personal fees from GSK, Novartis, Boehringer, AstraZeneca, Circassia, Sanofi and Menarini. PC provided consultancy services for Boehringer Ingelheim, Johnson \& Johnson, GSK, Merck Sharp \& Dohme, AstraZeneca, Novartis, Teva, Chiesi, and Sanofi; has served on advisory boards for Almirall, Boehringer Ingelheim, Johnson \& Johnson, GSK, AstraZeneca, Novartis, Teva, Chiesi, and Sanofi; has received lecture fees from Boehringer Ingelheim, Centocor, GSK, AstraZeneca, Novartis, Teva, Chiesi, Boston Scientific, and ALK-Abelló; and received industry-sponsored grants from Roche, Boston Scientific, Boehringer Ingelheim, Centocor, GSK, AstraZeneca, ALK-Abelló, Novartis, Teva, and Chiesi. PD reports personal fees from Astra Zeneca, Boehringer Ingelheim, Chiesi, GlaxoSmithKline, MundiPharma, Novartis, and Sanofi. During the past 3 years, PRB has received fees for lecturing/advisory boards from Astra Zeneca, Boehringer Ingelheim, Chiesi, GSK, Novartis, Pfizer, Teva, Vertex, Zambon. RK reports grants and personal fees from Air Liquide, Elivie, personal fees from Astra-Zeneca, Novartis, Boehringer Ingelheim. TC reports past collaborations (affiliation or financial interest or interest of any kind in the past 3 years) with AstraZeneca, GSK, Chiesi, Novartis and Boehringer-Ingelheim. YM reports past collaborations (as a national board member) with: Astra-Zeneca, Chiese, GSK, and Menarini. CB, CMS and MM report no conflicts of interest.

Patient and public involvement Patients and/or the public were not involved in the design, or conduct, or reporting or dissemination plans of this research.

Patient consent for publication Not required.
Ethics approval Ethics committee approval was not required.

Provenance and peer review Not commissioned; externally peer reviewed.

Data availability statement Data are available in a public, open access repository. The eo-Delphi protocol, questionnaires, and item lists are available via the Open Science Framework at DOl:10.5281/zenodo.3531475,https://osf.io/8f3aj/. The ranking dataset supporting the conclusions of this article is available in the Zenodo repository, DOI: 10.5281/zenodo.3531475; http://zenodo.org/record/3531475.

Open access This is an open access article distributed in accordance with the Creative Commons Attribution Non Commercial (CC BY-NC 4.0) license, which permits others to distribute, remix, adapt, build upon this work non-commercially, and license their derivative works on different terms, provided the original work is properly cited, appropriate credit is given, any changes made indicated, and the use is non-commercial. See: http://creativecommons.org/licenses/by-nc/4.0/.

\section{ORCID iD}

Carey Meredith Suehs http://orcid.org/0000-0002-2175-3496

\section{REFERENCES}

1 Glaab T, Vogelmeier C, Buhl R. Outcome measures in chronic obstructive pulmonary disease (COPD): strengths and limitations. Respir Res 2010;11:79.

2 Cazzola M, MacNee W, Martinez FJ, et al. Outcomes for COPD pharmacological trials: from lung function to biomarkers. Eur Respir J 2008;31:416-69.

3 FDA. Guidance for industry chronic obstructive pulmonary disease: developing drugs for treatment, 2007.

4 Jones P, Miravitlles M, van der Molen T, et al. Beyond FEV ${ }_{1}$ in COPD: a review of patient-reported outcomes and their measurement. Int $J$ Chron Obstruct Pulmon Dis 2012;7:697-709.

5 Cazzola M, Hanania NA, MacNee W, et al. A review of the most common patient-reported outcomes in COPD--revisiting current knowledge and estimating future challenges. Int J Chron Obstruct Pulmon Dis 2015;10:725-38.

6 Sinha IP, Smyth RL, Williamson PR. Using the Delphi technique to determine which outcomes to measure in clinical trials: recommendations for the future based on a systematic review of existing studies. PLoS Med 2011;8:e1000393.

7 GOLD. Global strategy for the diagnosis, management, and prevention of chronic obstructive pulmonary disease - 2018 Report, 2018.

8 Jouneau S, Dres M, Guerder A, et al. Management of acute exacerbations of chronic obstructive pulmonary disease (COPD). guidelines from the Société de pneumologie de langue Française (summary). Rev Mal Respir 2017;34:282-322.

9 Wedzicha JA, Miravitlles M, Hurst JR, et al. Management of COPD exacerbations: a European Respiratory Society/American Thoracic Society guideline. Eur Respir J 2017;49:1600791.

10 Niewoehner DE, Erbland ML, Deupree RH, et al. Effect of systemic glucocorticoids on exacerbations of chronic obstructive pulmonary disease. N Engl J Med Overseas Ed 1999;340:1941-7.

11 Bafadhel M, McKenna S, Terry S, et al. Blood eosinophils to direct corticosteroid treatment of exacerbations of chronic obstructive pulmonary disease: a randomized placebo-controlled trial. Am J Respir Crit Care Med 2012;186:48-55.

12 Bafadhel M, Davies L, Calverley PMA, et al. Blood eosinophil guided prednisolone therapy for exacerbations of COPD: a further analysis. Eur Respir J 2014;44:789-91.

13 Mathioudakis AG, Moberg M, Janner J, et al. Outcomes reported on the management of COPD exacerbations: a systematic survey of randomised controlled trials. ERJ Open Res 2019;5:00072-2019.

14 Brasel KJ, Lim HJ, Nirula R, et al. Length of stay: an appropriate quality measure? Arch Surg 2007;142:461.

15 Ruparel M, López-Campos JL, Castro-Acosta A, et al. Understanding variation in length of hospital stay for COPD exacerbation: European COPD audit. ERJ Open Res 2016;2:00034-2015.

16 Sivapalan P, Lapperre TS, Janner J, et al. Eosinophil-guided corticosteroid therapy in patients admitted to hospital with COPD exacerbation (CORTICO-COP): a multicentre, randomised, controlled, open-label, non-inferiority trial. Lancet Respir Med 2019;7:699-709.

17 Nelson WB. Recurrent events data analysis for product repairs, disease recurrences, and other applications. SIAM, 2003. 